\title{
Multiplicação in vitro e aclimatização de Melocactus sergipensis
}

\author{
In vitro multiplication and acclimatization of Melocactus sergipensis
}

D.0.I.: doi.org/10.30550/j.lil/2018.55.1/3

\author{
Bravo Filho, E. S. ${ }^{*}$; Marlucia, C. Santana ${ }^{2}$; Paulo, A. A. Santos ${ }^{2}$; \\ Adauto, S. Ribeiro ${ }^{3}$ \\ 1 Universidade Federal de Sergipe - UFS, Doutorado em Desenvolvimento e Meio Ambiente. Av. Marechal \\ Rondon s/n, C.E.P. 49.100-000, São Cristóvão-Sergipe, Brasil. \\ 2 Universidade Federal de Sergipe - UFS, Departamento de Biologia. Av. Marechal Rondon s/n, C.E.P. \\ 49.100-000, São Cristóvão-Sergipe, Brasil. \\ 3 Universidade Federal de Sergipe - UFS, Departamento de Ecologia. Av. Marechal Rondon S/n, C.E.P. \\ 49.100-000, São Cristóvão-Sergipe, Brasil. \\ * Autor para contato: esbravof@gmail.com
}

\begin{abstract}
Resumo - Melocactus sergipensis é uma espécie recém-descoberta, endêmica do estado de Sergipe e criticamente ameaçada de extinção. O objetivo desta pesquisa foi estabelecer um protocolo para micropropagação e aclimatização de plantas de $M$. sergipensis. 0 experimento foi instalado em delineamento inteiramente casualizado, com quatro tratamentos, 10 repetições e em cada unidade experimental foi introduzido um explante. Os explantes foram obtidos de seções medianas do caule com aproximadamente $5 \mathrm{~mm}$. O meio nutritivo foi 1/2 sais de MS suplementado com $30 \mathrm{~g} \mathrm{~L}^{-1}$ de sacarose, gelificado com $7 \mathrm{~g} \mathrm{~L}^{-1}$ de ágar e com as seguintes concentrações de fitorreguladores: 6-benzilaminopurina (BAP) [0,0; 1,5; 3,0 e $6,0 \mathrm{mg} \mathrm{L}^{-1}$ ] e ácido naftalenoaético [ANA] [0,0;1,5;3,0 e $6,0 \mathrm{mg} \mathrm{L}^{-1}$ ] e as combinações BAP/ANA [0,0; $1,0 / 0,5 ; 2,0 / 1,0$ e 4,0/2,0 $\left.\mathrm{mg} \mathrm{L}^{-1}\right]$. Foram avaliadas as médias de (brotos por explante, altura do caule e diâmetro do caule) e a porcentagem de calogênese, enraizamento explantes, sobrevivência dos explantes e brotos e peso da matéria fresca. Os dados foram submetidos à análise de variância e as médias comparadas pelo teste de Tukey, $5 \%$ de significância. Não houve diferença significativa entre os tratamentos em relação a formação de brotos, calos, sobrevivência dos brotos e explantes, altura do caule, diâmetro do caule, e peso matéria fresta. A suplementação no meio nutritivo com 1,0/0,5 BAP/ANA mg L-1 promoveu maior formação de brotos. Durante a fase ex vitro $70 \%$ dos brotos normais e $0,1 \%$ dos brotos hiperídricos sobreviveram.
\end{abstract}

Palavras-chave: Cabeça-de-frade; Cultivo ex vitro; Fitorreguladores; Micropropagação.

- Abstract - Melocactus sergipensis is a newly discovered species, endemic to the state of Sergipe and critically endangered. The objective of this research was to establish a protocol for micropropagation and acclimation of $M$. sergipensis plants. The experimental setting was a completely randomized design, with four treatments, 10 replicates and in each experimental unit an explant was introduced. Explants were obtained from medial sections of the stem of approximately $5 \mathrm{~mm}$. The nutrient medium was $1 / 2$ salts of $\mathrm{MS}$ supplemented with $30 \mathrm{~g} \mathrm{~L}^{-1}$ of

Ref. bibliográfica: Bravo Filho, E. S., Marlucia, C. S., Santos, A. A., Ribeiro, A. S. (2018). Multiplicaç ão in vitro e aclimatização de Melocactus sergipensis. Lilloa 55 (1): 26-36.

> Recibido: 14/02/18 - Aceptado: 11/05/18

$>$ URL de la revista: http://lilloa.lillo.org.ar

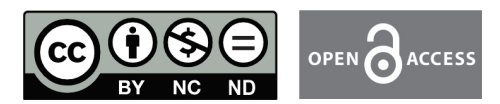

> Algunos derechos reservados. Esta obra está bajo una Licencia Creative Commons Atribución - No Comercial - Sin Obra Derivada 4.0 Internacional. 
sucrose, gelled with $7 \mathrm{~g} \mathrm{~L}^{-1}$ of agar and with the following concentrations of phytoregulators: 6-benzylaminopurine (BAP] [0,0; 1,5; 3,0 and $\left.6,0 \mathrm{mg} \mathrm{L}^{-1}\right]$ and naphthalenoacetic acid (ANA) $\left[0,0 ; 1,5 ; 3,0\right.$ and $\left.6,0 \mathrm{mg} \mathrm{L}^{-1}\right]$ and the BAP/ANA combinations $[0,0 ; 1,0 / 0,5 ; 2,0 / 1,0$ and $4,0 / 2,0 \mathrm{mg} \mathrm{L}^{-1}$ ]. The mean values of [shoots per explant, stem height and stem diameter] and percentage of calogenesis, rooting explants, survival of explants and shoots and weight of fresh matter were evaluated. Data were submitted to analysis of variance and means were compared by the Tukey test, $5 \%$ significance. There were no significant differences between treatments in relation to bud formation, callus, shoot and bud survival, stem height, stem diameter, and cracked weight. Nutritional supplementation with 1,0/0,5 BAP/ANA mg L-1 promoted a higher shoot formation. During the ex vitro phase, $70 \%$ of the normal shoots and $0,1 \%$ of the hyperhydric shoots survived.

Keywords: Turk's cap cactus; ex vitro culture; Phytoregulators; Micropropagation.

\section{INTRODUC̣ÃO}

Estudos sobre propagação são fundamentais para a conservação de cactos, especialmente para espécies que apresentam limitações reprodutivas a exemplo de dormência, baixos percentuais de germinação, ausência de brotações, crescimento lento e que necessitam de vários anos para atingir a fase reprodutiva (Marchi, 2016). Além disso, fatores como o extrativismo e a ocupação do solo para introdução de atividades agropastoris e imobiliária vem provocando redução dos habitats e consequentemente perda de diversidade genética e pode comprometer a sobrevivência das espécies [Plano Nacional Para conservação das Cactaceae [PAN] (2011); Livro Vermelho da Flora do Brasil [LVFB] (2013); Marchi, 2016; Silva e Ferreira, 2016].

As espécies do gênero Melocactus (LINK \& OTTO - Cactaceae), são popularmente conhecidas por cabeça-de-frade, coroa-de-frade, aleija-cavalo e tamborete-de-sogra (Bravo Filho, Santana, Santos, Ribeiro, 2018). Gênero composto por 38 espécies distribuídas desde a América Central, Caribe, Andes, Amazônia e Nordeste do Brasil, com exceção do Maranhão. O Brasil é o centro mundial de diversidade deste táxon, pois da totalidade de espécies que ocorre mundialmente, $23(60,5 \%)$ são nativas do Brasil, e destas 21 (55,2\%) são endêmicas (Resende, LimaBrito, Santana, 2010; [PAN], (2011); Zappi, Taylor, Santos, Larocca, 2015).

No estado de Sergipe, Nordeste do Brasil, ocorrem 26 espécies de Cactaceae e destas, uma é endêmica, seis exóticas e, apenas, o gênero Melocactus possui espécies ameaçadas de extinção, são elas: o Melocactus violaceus Pfeiff listado com o status de Vulnerável (VU) segundo Ministério do Meio Ambiente (Ministério do Meio Ambiente [MMA] (2014), Braun, Machado, Taylor, Zappi, 2017; Bravo Filho et al., 2018] e o Melocactus sergipensis N.P. Taylor \& M.V. Meiado, espécie rupícola descoberta em 2014, é atualmente a única espécie da família Cactaceae endêmica do estado e encontra-se Criticamente Ameaçada de Extinção (CR) (Taylor, Meiado, Bravo Filho, Zappi, 2014; Bravo Filho, Ribeiro, Sobral, 2015; Zappi et al. 2015; Convention on International Trade in Endangered Species of Wild Fauna and Flora [CITES], 2016).

O gênero Melocactus apresenta algumas limitações propagativas, a exemplo da ausência de emissão de brotos e ramificações, reprodução natural exclusivamente por sementes, crescimento lento, além da fase reprodutiva que ocorre por volta dos dez anos com o surgimento do cefálio (Hughes, 2009; Cruz, 2011; Coelho, Júnior, Nascimento, 2015).

As espécies do gênero Melocactus são coletadas indiscriminadamente para abastecer o comércio de plantas nativas, atividade que restringe cada vez mais a distribuição das espécies desse gênero, pois desconsidera o seu ritmo de resiliência e o alto grau de endemismo. As espécies coletadas são utilizadas na ornamentação, no místico-cultural, fabricação de alimentos (doces e ensopados) e objetos (enchimento para cangalha e almofadas), remédios caseiros e como forragem para ruminantes (Andrade, 2008; Hughes, 2009; PAN, 2011; Lucena, et al., 2012; Santos, Santos, Coutinho, Maoura, Antonio, 
2013; Neto, Castro Filho, Araújo, 2015; Bravo Filho et al., 2018).

Assim, uma alternativa adequada a conservação é a micropropagação, visto tratar-se de recurso genético endêmico e criticamente ameaçado de extinção, uma vez que essa técnica possibilita a multiplicação de plantas em grande quantidade, livre de patógenos, em menor intervalo de tempo, preserva as características genéticas e pode ser aplicada em qualquer estação do ano (Resende et al., 2010; Marchi, 2016; Lopes, Dias, Silveira, Rodrigues, Pasqual, 2017).

A aplicação de reguladores de crescimento tem sido muito utilizada na cultura de tecidos vegetais com o intuito de induzir organogênese, dentre estes, os mais utilizados são a citocinina, 6-benzilaminopurina (BAP), para a formação de brotos e auxina, ácido naftalenoacético (ANA), pelo efeito fisiológico no crescimento vegetal e no enraizamento, ou balanceando os dois reguladores (Piassi e Piassi, 2016; Silva e Ferreira, 2016). O objetivo desta pesquisa foi estabelecer um protocolo para micropropagação e aclimatização de brotos de Melocactus sergipensis.

\section{MATERIAL E MÉTODOS}

\section{LOCALIZAÇÃO DA ÁREA DE COLETA}

A espécie estudada ocorre no município de Simão Dias (Fig. 1), situado na região Sul do estado de Sergipe e possui uma área com aproximadamente 564,7 km². Confronta-se ao Norte com os municípios de Pinhão e Pedra Mole, ao Leste com Macambira e Lagarto, ao Sul com Riachão do Dantas e Lagarto e a Oeste com Tobias Barreto, Poço Verde e o estado da Bahia. A formação vegetal predominante é a Caatinga, clima semiárido, temperatura média de $23,7^{\circ} \mathrm{C}$ e índice pluviométrico médio anual de $845,5 \mathrm{~mm}$ (Silva e Silva, 2006; Secretaria de Estado do Meio Ambiente e dos Recursos Hídricos [SEMA$\mathrm{RH}$ ], 2012; Instituto Brasileiro de Geografia e Estatística [IBGE], 2016).

\section{MicropROPAGAÇÃO}

Para indução da multiplicação in vitro de M. sergipensis foram utilizadas plantas pré- estabelecidas in vitro com 120 dias após a germinação, oriundas de sementes obtidas de matrizes mantidas em casa de vegetação no Departamento de Biologia/CCBS da Universidade Federal de Sergipe. As plantas utilizadas possuíam de 0,8-1,2 cm de comprimento por 4-6 mm de diâmetro (Fig. 2A) e cultivadas em meio MS (Murashige e Skoog, 1962) com metade da concentração de sais, suplementado com $30 \mathrm{~g} \mathrm{~L}^{-1}$ de sacarose e gelificado com $7 \mathrm{~g} \mathrm{~L}^{-1}$ de ágar. $\mathrm{O} \mathrm{pH}$ foi ajustado para 5,8, inserido $1,5 \mathrm{~g} \mathrm{~L}^{-1} \mathrm{de}$ carvão ativado e autoclavado por $15 \mathrm{~min}$ sob pressão de $1,2 \mathrm{~atm}$. e temperatura $121^{\circ} \mathrm{C}$.

Foram avaliadas diferentes concentrações e combinações de reguladores de crescimento adicionados ao meio de cultura. As seguintes concentrações foram suplementadas ao meio: 6-benzilaminopurina (BAP: 0,0; 1,5; 3,0 e 6,0 mg L-1), ácido naftaleno acético (ANA: 0,0; 1,5; 3,0 e 6,0 $\mathrm{mg} \mathrm{L}^{-1}$ ) e diferentes balanços de BAP/ANA na proporção $2: 1$ : $(0,0 ; 1,0 / 0,5 ; 2,0 / 1,0$ e 4,0/2,0 mg $\left.\mathrm{L}^{-1}\right)$. O meio nutritivo utilizado na micropropagação foi suplementado com $30 \mathrm{~g} \mathrm{~L}^{-1} \mathrm{de}$ sacarose, geleificado com $7 \mathrm{~g} \mathrm{~L}^{-1}$ de ágar, $\mathrm{o}$ $\mathrm{pH}$ foi ajustado para 5,8, inserido $1,5 \mathrm{~g} \mathrm{~L}^{-1}$ de carvão ativado e autoclavado por $15 \mathrm{mi}$ nutos sob pressão de 1,2 atm. e temperatura de $121^{\circ} \mathrm{C}$.

Para obtenção dos explantes, as plantas foram seccionadas (Fig. 2A), os fragmentos medianos obtidos (Fig. 2B) foram divididos longitudinalmente em dois segmentos (Fig. 2C) com aproximadamente $5 \mathrm{~mm}$ de comprimento. Os explantes foram inoculados em tubos de ensaios contendo $10 \mathrm{~mL}$ de meio de cultura.

\section{DELINEAMENTO EXPERIMENTAL E ANÁLISE ESTATÍSTICA}

O delineamento experimental foi o inteiramente casualizado composto de quatro tratamentos (controle, BAP, ANA, e BAP/ ANA), dez repetições e em cada unidade experimental foi inserido um explante. A avaliação foi semanal e, após quatro meses de cultivo in vitro, foram analisadas as variáveis brotação (MB), altura do caule (MAC), 


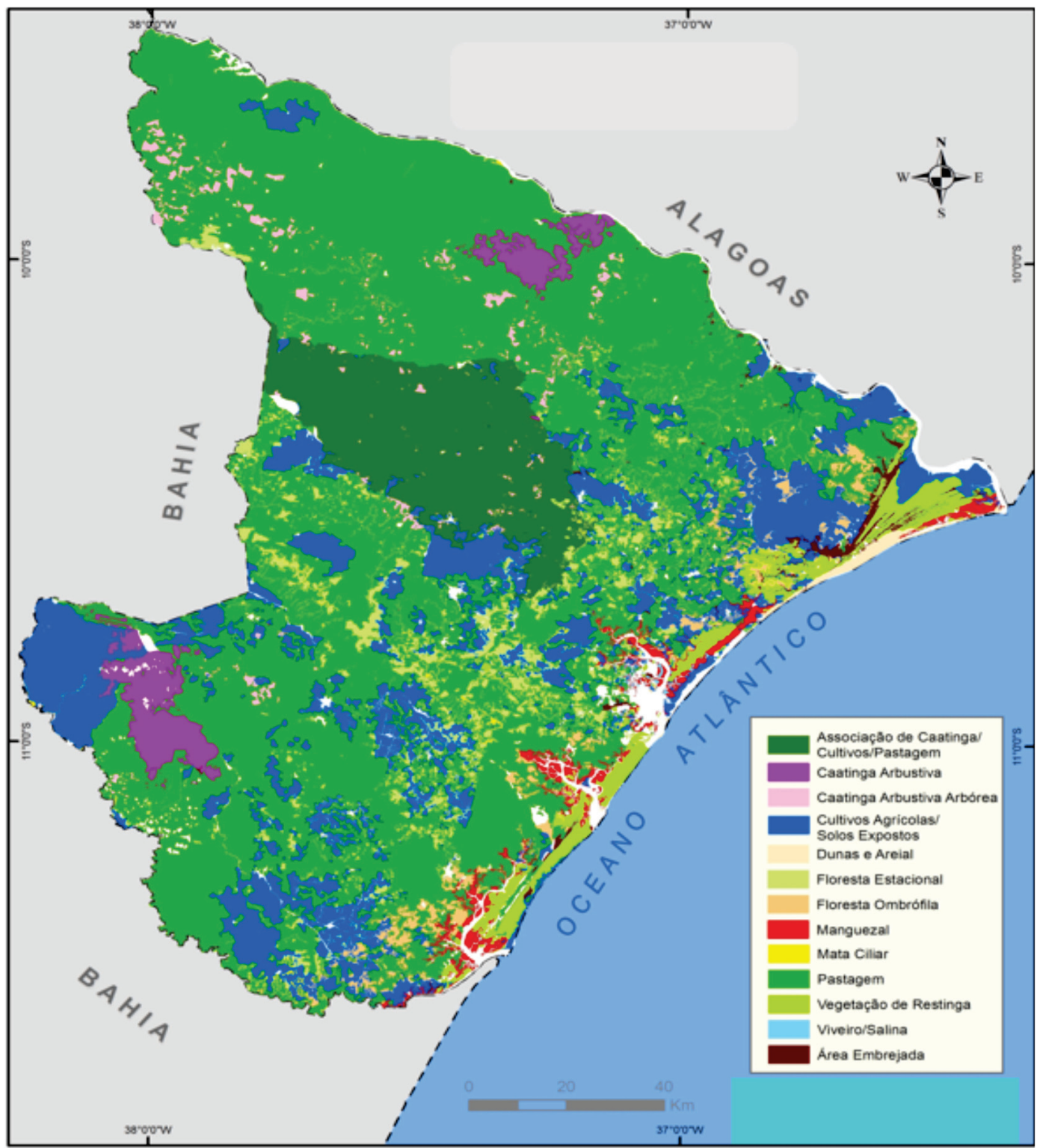

Fig. 1. Localização do município de Simão Dias no estado de Sergipe, local de ocorrência do M. sergipensis. Fonte: Modificado do Observatório de Sergipe [2016].

diâmetro do caule (MDC), indução de calo $(\% \mathrm{C})$, enraizamento explantes (\%RE), sobrevivência dos explantes (\%SE), sobrevivência dos brotos $(\% \mathrm{SB})]$, e peso da matéria fresca (PMF). Os dados foram submetidos à análise de variância (ANOVA) e as medias comparadas através do teste de Tukey a 5\% de significância.

\section{CONDIÇÃo DE CULTIVO}

As culturas foram mantidas em câmara incubadora tipo B.O.D., por cinco dias no escuro em temperatura controlada de $26 \pm 1$ ${ }^{\circ} \mathrm{C}$. Após esse período os experimentos foram mantidos em sala de crescimento por mais 115 dias, submetidos a um fotoperíodo de 16 $\mathrm{h}$ com intensidade luminosa de $45 \mu \mathrm{mol} \mathrm{m}{ }^{2} \mathrm{~s}^{-}$ 


\section{Corte transversal Corte longitudinal $\quad$ Explantes}

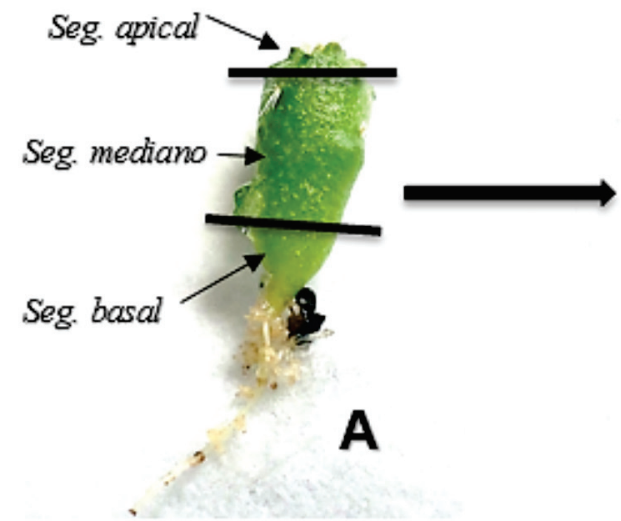



B

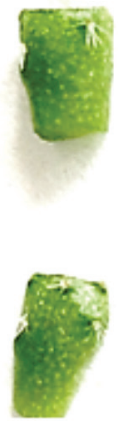

C

Fig. 2. Procedimento utilizado na produção de explantes em plantas de $M$. sergipensis com 120 dias pós-inoculação. A] Remoção dos segmentos apical e basal. B] Corte longitudinal no segmento mediano. C) explantes prontos para inoculação. Fonte: Bravo Filho (2017).

1, fornecida por lâmpadas fluorescentes de $40 \mathrm{~W}$ e temperatura média de $28 \pm 3^{\circ} \mathrm{C}$.

\section{ACLIMATIZAÇÃO}

Após 120 dias do cultivo in vitro, os brotos obtidos por meio da micropropagação, medindo $0,3-2,3 \mathrm{~cm}$ de comprimento por 0,3-1,4 cm de diâmetro e peso médio de $0,43 \mathrm{~g}$, foram extraídos dos explantes e submetidos ao processo de aclimatização.

Posteriormente a excisão, os brotos foram lavados em água corrente e transplantados para recipientes de polipropileno transparente com capacidade para $250 \mathrm{~mL}$, contendo $170 \mathrm{~g}$ de substrato (solo franco siltoso), umedecido com $20 \mathrm{~mL}$ de água e mantidos em sala de crescimento por mais 30 dias e, em seguida, transferidos para a casa de vegetação. Foram utilizados 20 recipientes, e inseridas três plantas por unidade experimental. A irrigação foi realizada uma vez por semana.

\section{RESULTADOS E DISCUSSÃO}

MULTIPLICAÇÃO IN VITRO DE BROTOS

DE M. SERGIPENSIS EM DIFERENTES CONCENTRAÇÕES DE BAP

Observa-se por meio da Tab.1, que os resultados obtidos e comparados a través do teste de Tukey a 5\% de significância, demostram que não houve diferença significativa entre as variáveis analisadas, porém, o tratamento composto por $6 \mathrm{mg} \mathrm{L}^{-1}$ de BAP apresentou a maior média de brotação em relação as outras concentrações. Em todos os tratamentos, os explantes e brotos apresentaram taxa de sobrevivência superior a $80 \%$ (Asmar, Resende, Andrade, Morais, Luiz, 2012) e Morais, Asmar, Luiz (2014) obtiveram resultados também superiores a $80 \%$ de sobrevivência de brotos de erva cidreira [Lippia alba (Mill.) N.E. Brown] e hortelãpimenta (Mentha $x$ piperita L.) utilizando as concentrações de $1,5 \mathrm{mg} \mathrm{L}^{-1} \mathrm{e} 4 \mathrm{mg} \mathrm{L}^{-1}$ de BAP respectivamente.

A suplementação com BAP em concentração de $6 \mathrm{mg} \mathrm{L}^{-1}$, apesar de não resultar em diferença significativa, apresentou os maiores valores quando comparado às demais variáveis analisadas, a exemplo da média de brotação (MB) que variou de 0,2-0,6 brotos (Tab. 1), porcentagem de enraizamento por explantes (\%RE) de 0,20-0,60 (Tab. 1), média altura do caule (MAC) de 0,48-0,73 cm (Tab. 1) e média peso matéria fresca (PMF) de 0,08-1,05 g (Tab. 1). Em contrapartida, brotos com maiores médias do diâmetro do caule $(0,70 \mathrm{~mm})$ foram obtidos no meio nu- 
tritivo suplementado com 3,0 $\mathrm{mg} \mathrm{L}^{-1}$ de BAP (Tab.1).

Esses resultados divergem dos obtidos por Asmar et al. (2012), ao pesquisarem a concentração de hormônio BAP mais adequada para indução da brotação de erva cidreira. Esses autores concluíram que a suplementação com 1,5 mg L-1 de BAP, estimulou maior número de brotação. No entanto para a espécie estudada nesta pesquisa, na concentração de $6 \mathrm{mg} \mathrm{L}-1$ de BAP (Tab. 1) obteve-se os maiores valores.

Lopes et al. (2017) estudaram diversas concentrações de BAP, com intuito de determinar a concentração mais eficaz na proliferação e desenvolvimento de brotos pitaia (Hylocereus undatus - Cactaceae), observaram que a concentração de $1,0 \mathrm{mg} \mathrm{L}^{-1}$ de BAP induziu um maior número de brotos. Resultados similares foram observados por Jardim, Sampaio, Costa, Gonçalves, Brandão (2010), ao estudarem o efeito de diferentes reguladores de crescimento na regeneração in vitro de pau-rosa (Aniba rosaeodora Ducke), uma vez que, obtiveram maior número de brotação por explantes na contração de $4 \mathrm{mg} \mathrm{L}^{-1}$ de BAP.

\section{MULTIPLICAÇÃO IN VITRO DE BROTOS DE M. SERGIPENSIS EM DIFERENTES CONCENTRAÇÕES DE ANA}

Os resultados obtidos e comparados através do teste de Tukey a 5\% de probabilidade (Tab. 2), demostram que não houve diferença significativa no número brotação por explantes, porém, o balanceamento hormonal de ANA, proporcionou elevação na média das principais variáveis analisadas. Em todas as concentrações, os brotos apresentaram 100\% de sobrevivência. Já em relação a os explantes a taxa de sobrevivência foi superior a $80 \%$ (Tab. 2). Resultados semelhantes foram obtidos por Oliveira, Freire, Aloufa (2016), ao estudarem proliferação de brotos de mangabeira, pois obtiveram taxa de sobrevivências acima de 90\% em concentração de $0,5 \mathrm{mg} \mathrm{L}^{-1}$ de ANA.

A suplementação com o hormônio ANA na concentração de $1,5 \mathrm{mg} \mathrm{L}^{-1}$, promove maiores resultados quando comparado às demais variáveis analisadas, a exemplo da média de brotação (MB) que variou de 0,10,6 brotos (Tab. 2), média da altura do caule (MAC) de 0,48-1,42 cm (Tab. 2) e peso médio da matéria fresca (PMF) de 0,08-0,60 g (Tab. 2). Em relação à variável porcentagem de enraizamento por explantes (\%RE), a utilização de $3 \mathrm{mg} \mathrm{L}^{-1}$ propiciou maiores resultados variando de 0,40-0,80 explante com raiz (Tab. 2). Estes resultados convergem com os obtidos por Jardim et al. (2010), em pesquisa sobre a ação dos fitormônios na indução de brotos de pau-rosa cultivados in vitro. Porém, nas concentrações de $3 \mathrm{mg} \mathrm{L}^{-1}$ e $6 \mathrm{mg} \mathrm{\textrm {L } ^ { - 1 }}$ de ANA foi observado decréscimo no número de brotos de $M$. sergipensis (Tab. 2) em relação ao controle.

Nesta pesquisa, a medida que elevou-se a concentração de ANA no meio nutritivo a partir de $1,5 \mathrm{mg} \mathrm{L}-1$, ocorreu decréscimo na altura do caule (Tab. 2), tais resultados convergem como os obtidos por Brum, Silva, Pasqual (2002) e Oliveira et al. (2016), ao estudarem efeito de diferentes concentrações de BAP e ANA na multiplicação de brotos in vitro da figueira (Ficus carica L.) e mangabeira (Hancornia speciosa Gomes) respectivamente, tais autores observaram decréscimo na altura dos brotos das espécies estudadas, sendo a redução inversamente proporcional às dosagens do hormônio ANA quando comparado ao controle.

\section{MULTIPLICAÇÃO IN VITRO DE BROTOS}

DE M. SERGIPENSIS EM DIFERENTES

\section{CONCENTRAÇÕES COMBINANDO BAP/ANA}

Os resultados obtidos e comparados através do teste de Tukey 5\% de probabilidade (Tab. 3), demostram que não houve diferença significativa no número brotação por explantes, porém, houve diferença significativa na média de enraizamento por explantes. Em todos os tratamentos os explantes e brotos apresentaram taxa de sobrevivência superior a 80\%. Esses resultados convergem com os obtidos por Morais et al. (2014), ao pesquisarem a ação dos reguladores de crescimento vegetal no cultivo in vitro de hortelã-pimenta (Mentha x piperita 
L.), observaram aumento significativo da sobrevivência dos explantes, principalmente na concentração de $4 \mathrm{mg} \mathrm{L}^{-1}$ de BAP, independente das doses de ANA e $\mathrm{GA}_{3}$.

A aplicação combinando BAP/ANA (Tab. 3) na proporção $\left(1 / 0,5 \mathrm{mg} \mathrm{L}^{-1}\right)$ apresenta diferença significativa apenas na variável porcentagem de enraizamento por explante (\%RE) 0,0-0,80 (Tab. 3), já em relação as demais variáveis não houve diferença significativa, contudo esse balanço hormonal promoveu maiores resultados na média de brotação (MB)40-70\% (Tab. 3), percentual de sobrevivência dos explantes (\%SE) 80$100 \%$, média altura do caule (MAC) 0,48$1,01 \mathrm{~cm}$ (Tab. 3), média diâmetro do caule (MDC) $0,45-0,69 \mathrm{~mm}$ e peso matéria fresca (PMF) 0,08-0,55 g.

Tabela 1. Desenvolvimento de brotos de $M$. sergipensis in vitro com suplementação de BAP 120 dias após inoculação. Média de brotação $(\mathrm{MB})$, porcentagem de calo $[\% \mathrm{C}$ ), porcentagem de enraizamento por explantes [\%RE], porcentagem de sobrevivência explantes [\%SE], porcentagem de sobrevivência brotos (\%SB], média altura do caule (MAC), média diâmetro do caule (MDC) e peso matéria fresca (PMF).

\begin{tabular}{lcccccccc}
\hline & MB & \%C & \%RE & \%SE & \%SB & $\begin{array}{c}\text { MAC } \\
(\mathrm{cm})\end{array}$ & $\begin{array}{c}\text { MDC- } \\
(\mathrm{mm})\end{array}$ & $\begin{array}{c}\text { PMF } \\
(\mathrm{g})\end{array}$ \\
\hline Controle & $0,50 \mathrm{a}$ & $0,10 \mathrm{a}$ & $0,20 \mathrm{a}$ & $0,80 \mathrm{a}$ & $1,00 \mathrm{a}$ & $0,48 \mathrm{a}$ & $0,45 \mathrm{a}$ & $0,08 \mathrm{a}$ \\
\hline BAP $\left(1,5 \mathrm{mg} \mathrm{L}^{-1}\right)$ & $0,30 \mathrm{a}$ & $0,10 \mathrm{a}$ & $0,30 \mathrm{a}$ & $0,80 \mathrm{a}$ & $1,00 \mathrm{a}$ & $0,70 \mathrm{a}$ & $0,55 \mathrm{a}$ & $0,19 \mathrm{a}$ \\
\hline $\operatorname{BAP}\left(3,0 \mathrm{mg} \mathrm{L}^{-1}\right)$ & $0,20 \mathrm{a}$ & $0,00 \mathrm{a}$ & $0,40 \mathrm{a}$ & $0,90 \mathrm{a}$ & $1,00 \mathrm{a}$ & $0,60 \mathrm{a}$ & $0,70 \mathrm{a}$ & $0,17 \mathrm{a}$ \\
\hline BAP $\left(6,0 \mathrm{mg} \mathrm{L}^{-1}\right)$ & $0,60 \mathrm{a}$ & $0,00 \mathrm{a}$ & $0,60 \mathrm{a}$ & $0,80 \mathrm{a}$ & $1,00 \mathrm{a}$ & $0,73 \mathrm{a}$ & $0,50 \mathrm{a}$ & $1,05 \mathrm{a}$ \\
\hline
\end{tabular}

Obs.: Letras iguais indicam que, ao nível de $5 \%$ de significância, não há diferença entre as médias.

Tabela 2. Desenvolvimento de brotos de $M$. sergipensis in vitro com suplementação de ANA 120 dias após inoculação. Média de brotação $(\mathrm{MB})$, porcentagem de calo $[\% \mathrm{C}]$, porcentagem de enraizamento por explantes [\%RE], porcentagem de sobrevivência explantes (\%SE), porcentagem de sobrevivência brotos (\%SB), média altura do caule (MAC), média diâmetro do caule (MDC) e peso matéria fresca (PMF).

\begin{tabular}{lcccccccc}
\hline & MB & \%C & \%RE & \%SE & \%SB & $\begin{array}{c}\text { MAC } \\
(\mathrm{cm})\end{array}$ & $\begin{array}{c}\text { MDC- } \\
(\mathrm{mm})\end{array}$ & $\begin{array}{c}\text { PMF } \\
(\mathrm{g})\end{array}$ \\
\hline Controle & $0,50 \mathrm{a}$ & $0,10 \mathrm{a}$ & $0,20 \mathrm{a}$ & $0,80 \mathrm{a}$ & $1,00 \mathrm{a}$ & $0,48 \mathrm{a}$ & $0,45 \mathrm{a}$ & $0,08 \mathrm{a}$ \\
\hline ANA $\left(1,5 \mathrm{mg} \mathrm{L}^{-1}\right)$ & $0,60 \mathrm{a}$ & $0,10 \mathrm{a}$ & $0,40 \mathrm{a}$ & $0,80 \mathrm{a}$ & $1,00 \mathrm{a}$ & $1,42 \mathrm{a}$ & $1,05 \mathrm{a}$ & $0,60 \mathrm{a}$ \\
\hline ANA $\left(3,0 \mathrm{mg} \mathrm{L}^{-1}\right)$ & $0,10 \mathrm{a}$ & $0,00 \mathrm{a}$ & $0,80 \mathrm{a}$ & $1,00 \mathrm{a}$ & $1,00 \mathrm{a}$ & $0,70 \mathrm{a}$ & $0,50 \mathrm{a}$ & $0,60 \mathrm{a}$ \\
\hline ANA $\left(6,0 \mathrm{mg} \mathrm{L}^{-1}\right)$ & $0,10 \mathrm{a}$ & $0,00 \mathrm{a}$ & $0,40 \mathrm{a}$ & $1,00 \mathrm{a}$ & $1,00 \mathrm{a}$ & $0,60 \mathrm{a}$ & $0,50 \mathrm{a}$ & $0,60 \mathrm{a}$ \\
\hline
\end{tabular}

Obs.: Letras iguais indicam que, ao nível de $5 \%$ de significância, não há diferença entre as médias.

Tabela 3. Desenvolvimento de brotos de M. sergipensis in vitro com suplementação de BAP/ ANA 120 dias após inoculação. Média de brotação (MB), porcentagem de calo $(\% \mathrm{C})$, porcentagem de enraizamento por explantes [\%RE], porcentagem de sobrevivência explantes [\%SE], porcentagem de sobrevivência brotos (\%SB], média altura do caule (MAC), média diâmetro do caule $(\mathrm{MDC})$ e peso matéria fresca (PMF).

\begin{tabular}{lcccccccc}
\hline & MB & \%C & \%RE & \%SE & \%SB & $\begin{array}{c}\text { MAC } \\
(\mathrm{cm})\end{array}$ & $\begin{array}{c}\text { MDC- } \\
(\mathrm{mm})\end{array}$ & $\begin{array}{c}\text { PMF } \\
(\mathrm{g})\end{array}$ \\
\hline Controle & $0,50 \mathrm{a}$ & $0,10 \mathrm{a}$ & $0,20 \mathrm{ab}$ & $0,80 \mathrm{a}$ & $1,00 \mathrm{a}$ & $0,48 \mathrm{a}$ & $0,45 \mathrm{a}$ & $0,08 \mathrm{a}$ \\
\hline BAP/ANA $\left(1 / 0,5 \mathrm{mg} \mathrm{L}^{-1}\right)$ & $0,70 \mathrm{a}$ & $0,10 \mathrm{a}$ & $0,80 \mathrm{a}$ & $1,00 \mathrm{a}$ & $1,00 \mathrm{a}$ & $1,01 \mathrm{a}$ & $0,69 \mathrm{a}$ & $0,55 \mathrm{a}$ \\
\hline BAP/ANA $\left(2 / 1 \mathrm{mg} \mathrm{L}^{-1}\right)$ & $0,40 \mathrm{a}$ & $0,00 \mathrm{a}$ & $0,80 \mathrm{a}$ & $1,00 \mathrm{a}$ & $1,00 \mathrm{a}$ & $0,50 \mathrm{a}$ & $0,50 \mathrm{a}$ & $0,06 \mathrm{a}$ \\
\hline BAP/ANA $\left(4 / 2 \mathrm{mg} \mathrm{L}^{-1}\right)$ & $0,40 \mathrm{a}$ & $0,00 \mathrm{a}$ & $0,00 \mathrm{~b}$ & $1,00 \mathrm{a}$ & $1,00 \mathrm{a}$ & $0,97 \mathrm{a}$ & $0,60 \mathrm{a}$ & $0,44 \mathrm{a}$ \\
\hline
\end{tabular}

Obs.: Letras iguais indicam que, ao nível de 5\% de significância, não há diferença entre as médias. 
Resultados semelhantes foram obtidos por Piassi e Piassi (2016), ao pesquisarem concentrações combinando $1,0 / 0,05 \mathrm{e}$ 2,0/0,1 mgL-1 de BAP/ANA, na indução do crescimento dos explantes de alface (Lactuca sativa L.), obtiveram maiores resultados tanto para o diâmetro caulinar, com maior quantidade de explantes com presença de calogênese variando entre 50 e 100\% respectivamente.

Resultados divergentes formam obtidos por Brum et al. (2002), que observaram decréscimo na brotação de plantas de figueira (Ficus carica L.) quando comparado com o controle nos tratamentos contendo suplementações combinadas dos hormônios BAP/ANA nas proporções avaliadas, contudo à medida que a concentração de ANA foi aumentada entre 0,05 a 2,0 $\mathrm{mg} \mathrm{L}^{-1}$, ocorreu crescimento linear das raízes.

\section{DESENVOLVIMENTO E ACLIMATIZAÇÃO DAS PLANTAS OBTIDAS IN VITRO}

Após nove dias de inoculação surgiu as primeiras radículas (Fig. 3A) e calogênese (Fig. 3B) em alguns explantes, no vigésimo terceiro dia iniciar-se o surgimento dos primeiros brotos (Figs. 3C e 3D) em todos os tratamentos e com maior número de bro- tação no meio nutritivo suplementado por 1,0/0,5 mg L-1 de BAP/ANA (Marchi, 2016), em estudo sobre micropropagação, constatou diferenciação em brotos axilares de Stephanocereus luetzelburgii após três semanas de cultivo in vitro.

No tratamento composto por $6 \mathrm{mg} \mathrm{L}^{-1} \mathrm{de}$ BAP, $100 \%$ dos explantes que emitiram brotos desenvolveram também raízes (Fig. 3E). Os tratamentos compostos por 1,5 $\mathrm{mg} \mathrm{L}^{-1} \mathrm{de}$ BAP e 1,0/0,5 mg L-1 de BAP/ANA induziram múltiplas brotações (Figs. 3F e 3G).

Para a aclimatização, os brotos foram seccionados dos explantes (Fig. 4A) e introduzidos diretamente no substrato (Fig. 4B) sem passar pela etapa de enraizamento in vitro, ocorreu 70\% de sobrevivência para os brotos normais em condição ex vitro (Fig. 4C). Já, 99\% dos brotos hiperídricos (Figs. 4D e 4E) morreram nos primeiros oito dias pós-transplantação para condição ex vitro. Resultados semelhantes foram obtidos por Resende et al. (2010) e Marchi (2016), ao aplicarem o mesmo procedimento na aclimatização de brotos obtidos in vitro das espécies $M$. glaucescens e Stephanocereus luetzelburgii, respectivamente, pois obtiveram 93 e 100\% de sobrevivência para os brotos normais. Enquanto Dorneles e trivelin (2011), obtive-

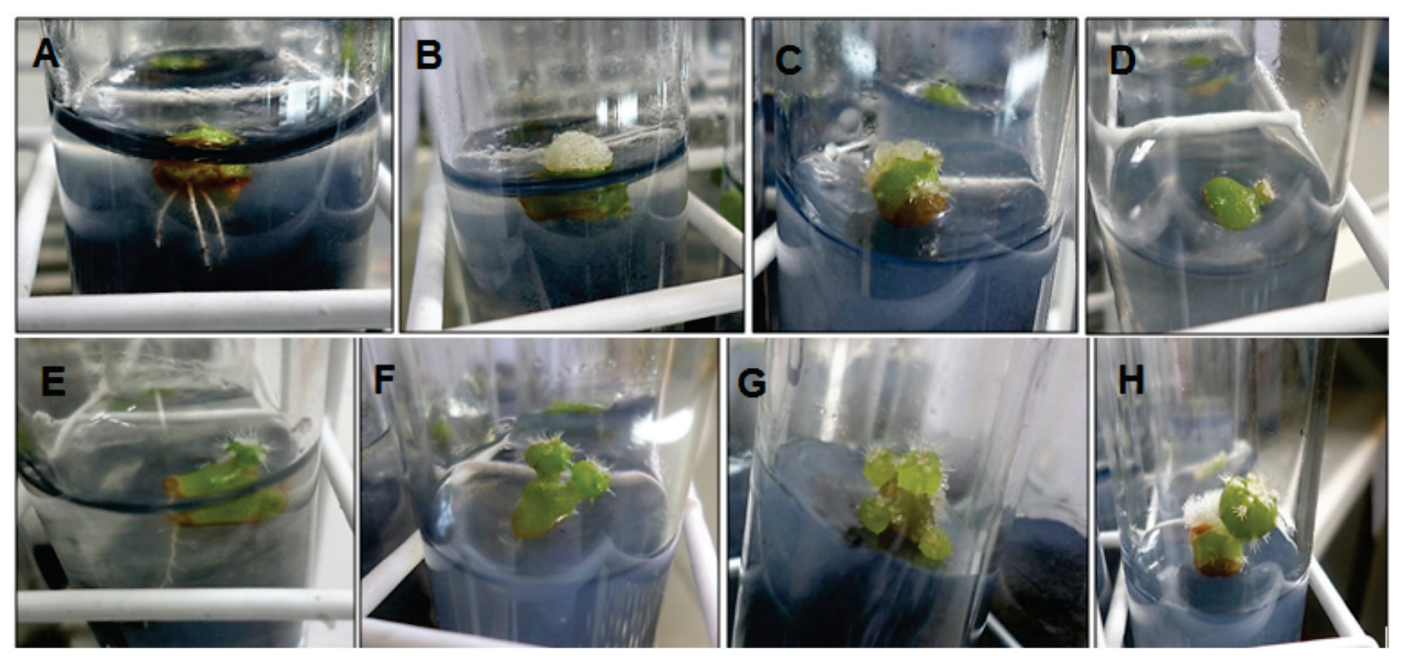

Fig. 3. Desenvolvimento dos brotos de M. sergipensis no intervalo de 120 dias. A) Surgimento das raízes no explante. B] Explante com calogênese. C) Surgimento dos primeiros brotos. D) Explante com broto, contudo sem raízes. E] Explante com broto e raiz. F e G) Explantes com múltiplos brotos. H] Broto normal ao fim das avaliações. Fonte: Bravo Filho (2017). 
ram 53\% de sobrevivência na aclimatização da orquídea Cattleya intermedia Graham ex Hook (Orchidaceae) obtidas por propagação in vitro.

\section{CONCLUSÕES}

Com base nos resultados, pode-se considerar que a suplementação balanceada de BAP/ANA no meio de nutritivo na propor-


Fig. 4. Etapas utilizadas para a aclimatização de plantas de M. sergipensis. A) Explantes com brotos. B] Brotos removidos dos explantes. C e D) Brotos transplantados para o substrato. E) Plantas após período de aclimatização. Fonte: Bravo Filho (2017-2018). 
ção de 1,0/0,5 mg L-1 proporcionou maior número de brotação durante a fase de multiplicação in vitro de $M$. sergipensis quando comparado aos demais tratamentos.

Para a aclimatização de brotos de $M$. sergipensis obtidos in vitro não foi necessária prévia indução in vitro do desenvolvimento do sistema radicular, pois $70 \%$ dos brotos que sobreviveram pós-transplantação para o substrato, todos desenvolveram o sistema radicular, por outro lado, os brotos hiperídricos 99\% morreram nos primeiros oito dias pós-transplantação para condição ex vitro.

\section{BIBLIOGRAFIA}

Andrade C. T. S. (2008). Cactos Úteis na Bahia: Ênfase do Semi-árido. USEB, Rio Grande do Sul.

Asmar S. A., Resende R. F., Araruna E. C., Morais T. P. e Luiz J. M. Q. (2012). Concentrações de BAP sobre a proliferação in vitro de brotos de Lippia alba [(Mill.] N. E. Brown]. Revista Brasileira de Plantas Medicinais 14: 149-153.

Braun P., Machado M., Taylor, N. P. e Zappi D. (2017). Melocactus violaceus. (amended version published in 2013) The IUCN Red List of Threatened Species 2017: e.T40925A121501259. Recuperado de http://dx.doi.org/10.2305/IUCN. UK.2017-3.RLTS.T40925A121501259. en. Acesso janeiro 2018.

Bravo Filho E. S., Ribeiro A. S. e Sobral I. S. (2015). Legislação Ambiental: Subsídio para a conservação das cactáceas nativas. In: M. J. Nascimento Soares, Org. Pesquisa Ambiental em Foco. Criação, Aracaju, pp. 199-210.

Bravo Filho, E. S., Santana, M. C., Santos, P. A. A. e Ribeiro, A. S. (2018). Levantamento etnobotânico da família Cactaceae no estado de Sergipe. Revista Fitos 12 (1): 41-53.

Brum G. R., Silva A. B. e Pasqual M. (2002). Efeito de diferentes concentrações de BAP E ANA na propagação in vitro da figueira (Ficus carica L.). Ciências agrotec., Lavras Edição Especial: 14031409.

Convention on International Trade in Endangered Species of Wild Fauna and Flora [CITES]. (2016). Cactaceae checklist. Recuperado de http://assets.kew.org/ files/CITES\%2OCactaceae\%2OChecklist\% 20Third\%20Edition.pdf. Acesso outubro 2016.
Coelho P. J. A., Júnior S. F. F. e Nascimento E. (2015). Coleta e conservação ex situ de cactáceas nativas do estado do CEARÁ. Gaia Scientia 9 (2): 183-192.

Cruz B. M. (2011). Estudos de longevidade e germinação em sementes de espécies do gênero Melocactus (Cactaceae) de Morro do Chapéu, Chapada Diamantina, Bahia. Dissertação (Dissertação de Mestrado), Universidade Federal de Feira de Santana, feira de Santana.

Dorneles L. T. e Trevelin V. (2011). Aclimatização e reintrodução de Cattleya intermedia Graham ex Hook (Orchidaceae) obtidas por propagação in vitro. Iheringia 66 (2): 167-174.

Hughes F. M. (2009). Ecologia reprodutiva e morfometria de Melocactus ernestii (Vaupel] no Município de Nova Itarana. (Dissertação de Mestrado), Universidade Estadual de Feira de Santana, feira de Santana.

Instituto Brasileiro de Geografia e Estatística [IBGE]. (2016). Cidades. Recuperado de http://www.cidades.ibge.gov.br/xtras/ perfil. php? lang=\&codmun=280020. Acesso outubro 2016.

Jardim L. S., Sampaio P. T. B., Costa S. S., Gonçalves C. Q. B. e Brandão H. L. M. (2010). Efeito de diferentes reguladores de crescimento na regeneração in vitro de pau-rosa (Aniba rosaeodora Ducke). Acta Amazônica 40 (2): 275-280.

Livro Vermelho da Flora do Brasil [LVFB]. (2013). Centro Nacional de Conservação da Flora (CNCFLORA). Recuperado de www.cncflora.jbrj.gov.br/arquivos/arqui. Acesso novembro 2017.

Lopes C. A., Dias G. M. G., Silveira F. A., Rodrigues F. A., Pio L. A. S. e Pasqual M. (2017). Propagação in vitro de pitaia vermelha. Plant Cell Culture \& Micropropagation 13 (1): 21-27.

Lucena C. M., Costa G. M., Sousa R. F., Carvalho T. K. N., Marreiros N. A., Alves C. A. B., Pereira D. D. e Lucena R. F. P. (2012). Conhecimento local sobre Cactáceas em comunidades rurais na mesorregião do sertão da Paraíba (Nordeste, Brasil). Universidade Federal da Paraíba 25 (3): 282-288.

Marchi M. N. G. (2016). Aspectos fisiológicos, anatômicos e moleculares da propagação e conservação in vitro de espécies de cactos endêmicos da $\mathrm{Ba}$ hia. Tese de Doutorado), Universidade Estadual de Feira de Santana, Feira de Santana.

Ministério do Meio Ambiente [MMA]. (2014). Caatinga. Recuperado de http://www. 
mma.gov.br/biomas/caatinga. Acesso dezembro 2017

Morais T. P., Asmar S. A. e Luiz J. M. Q. [2014]. Reguladores de crescimento vegetal no cultivo in vitro de Mentha $\mathrm{x}$ piperita L. Revista Brasileira de Plantas Medicinais 16 (2): 350-355.

Murashige T. e Skoog F. (1962). A revised medium for rapid growth and bioassays with tobacco tissue culture. Physiologia Plantarum 15: 473-497.

Neto J. A. S., Castro Filho E. S. e Araújo H. R. (2015). Potencial das cactáceas como alternativa alimentar para ruminantes no semiárido. Nutritime Revista Eletrônica 12: 4426-4434.

Oliveira K. S., Freire F. A. M.e Aloufa M. A. I. (2016). Efeito de 6-benzilaminopurina e ácido naftalenoacético sobre a propagação in vitro de Hancornia speciosa Gomes. Revista Floresta 46 (3): 335342 .

Piassi M., Piassi M. (2016).Otimização de protocolo para indução da calogênese in vitro em folhas cotiledonares de alface [Lactuca sativa L.]. Revista Intelletto 2: 135-142.

Plano de Ação Nacional para a Conservação das Cactáceas [PAN]. (2011). Daniela Zappi et al., Brasília: Instituto Chico Mendes de Conservação da Biodiversidade, ICMBio.
Resende, S. V., Lima-Brito, A. e Santana, J. R. (2010). Influência do substrato e do enraizamento na aclimatização de Melocactus glaucesceus Brining \& Brederoo propagação in vitro. Revista Ceres 57 (6): 803-809.

Santos P. T. S., Santos S. M., Coutrinho A. P., Moura G. S. S.e Antonino A. C. D. (2013). Telhado verde: desempenho do sistema construtivo na redução do escoamento superficial. Revista Ambiente Construido 13 (1): 161-174.

Secretaria de Estado do Meio Ambiente e dos Recursos Hídricos [ SEMARH]. [2012]. Florestas em Sergipe: Construindo uma política florestal. Aracaju.

Silva M. M. A. e Ferreira L. T. (2016). Cultivo in vitro de plantas e suas aplicações em Cactaceae. INSA, Campinas Grande.

Silva F. H. B. B. e Silva A. B. (2006). Levantamento de reconhecimento de solos e avaliação do potencial de terras para irrigação do município de Simão Dias, Sergipe. Embrapa- RJ Comunica Técnica 39: 1-10.

Taylor, N. P., Meiado, M. V., Bravo Filho, E. e Zappi, D. [2014). A new Melocactus from the Brazilian state of Sergipe. Bradleya 32: 99-104.

Zappi, D., Taylor, N., Santos, M. R. e Larocca, J. (2015). Cactaceae in Lista de Espécimes da Flora do Brasil. Recuperado de http://reflora.jbrj.gov.br/jabot/floradobrasil/FB1558. Acesso junho 2017. 